\title{
Effect of Chitosan and Liposome Nanoparticles as Adjuvant Codelivery on the Immunoglobulin G Subclass Distribution in a Mouse Model
}

\author{
Agus Haryono, ${ }^{1}$ Korrie Salsabila, ${ }^{2}$ Witta Kartika Restu, ${ }^{1}$ Sri Budi Harmami, ${ }^{1}$ and \\ Dodi Safari $^{2}$ \\ ${ }^{1}$ Research Center for Chemistry Indonesian Institute of Sciences, Kawasan Puspiptek Serpong, Tangerang, Indonesia \\ ${ }^{2}$ Eijkman Institute for Molecular Biology, Jakarta, Indonesia \\ Correspondence should be addressed to Dodi Safari; safari@eijkman.go.id
}

Received 17 February 2017; Revised 16 May 2017; Accepted 4 June 2017; Published 5 July 2017

Academic Editor: Kurt Blaser

Copyright (c) 2017 Agus Haryono et al. This is an open access article distributed under the Creative Commons Attribution License, which permits unrestricted use, distribution, and reproduction in any medium, provided the original work is properly cited.

\begin{abstract}
Background. We investigate the immunogenic properties of chitosan and liposome nanoparticles as adjuvant codelivery against a commercial pneumococcal conjugate vaccine (PCV) in an animal model. Methods. The chitosan and liposome nanoparticles were prepared by ionic gelation and dry methods, respectively. The PCV immunization was performed intradermally in the presence of adjuvants and booster injections which were given without an adjuvant. The Quil- ${ }^{\circledR}{ }^{\circledR}$ was used as a control adjuvant. The ELISA was performed to measure the antibodies against pneumococcal type 14 polysaccharide (Pn14PS). Results. The level of total antibodies against Pn14PS antigen was no different between the mouse groups with or without adjuvant codelivery. Codelivery of the PCV with chitosan nanoparticles as well as the Quil-A adjuvant elicited IgG1, IgG2a, IgG2b, and IgG3 antibodies. Meanwhile, codelivery of liposome nanoparticles elicited mainly IgG1 antibodies against the Pn14PS. Conclusions. The chitosan and liposome nanoparticles as adjuvant codelivery were successfully synthesized. These nanoparticles have different shapes in particle formation, liposome nanoparticle with their unilamellar shape and chitosan nanoparticles in large shape due to the aggregation of small-size particles. Codelivery of chitosan nanoparticles has more effect on the IgG subclass antibody production than that of liposome nanoparticles in a mouse model.
\end{abstract}

\section{Introduction}

Nanoparticles are increasingly used as adjuvant vaccine formulations due to their biocompatibility, ease of manufacture, and the opportunity to tailor their size, shape, and physicochemical properties [1]. Chitosan is a nontoxic, biocompatible, biodegradable, natural polysaccharide whose the molecular weight is between 3800 and 20,000 Daltons and the degree of deacetylation ranges from $60 \%$ to $100 \%$ [2]. Due to its useful properties, chitosan offers advantages for adjuvant and vaccine delivery systems $[3,4]$. Sáenz et al. reported that the chitosan formulation improves the immunogenicity of a peptide-based vaccine and was able to induce an immune response mediated by IgG2a [5]. Liposomes, sphere-shaped vesicles with particle sizes ranging from $30 \mathrm{~nm}$ to several micrometers consisting of one or more phospholipid bilayers, were characterized to deliver active molecules to the site actions, and at present, several formulations are in clinical use [6, 7]. Liposomes are structurally suitable to make nanoparticles biocompatible and offer a clinically proven, versatile platform for the further enhancement of pharmacological efficacy [8]. It was reported that a cationic liposome-forming lipid worked as potential adjuvants for protein subunit vaccines. This potential adjuvant also appeared to favour a stronger Th1 immune response [9].

In this present study, we investigate the immunogenic properties of chitosan and liposome nanoparticles as adjuvant codelivery against a commercial pneumococcal conjugate vaccine $(\mathrm{PCV})$ in an animal model. 


\section{Materials and Methods}

2.1. Adjuvants and Vaccine. Low molecular weight chitosan $\left(\mathrm{C}_{8} \mathrm{H}_{15} \mathrm{NO}_{6}\right)_{\mathrm{n}}$ powder with $75 \%$ degree of deacetylation and soybean phosphatidylcholine were purchased from Sigma-Aldrich, USA. The Quil-A saponin adjuvant was a gift from Dr. Erik B. Lindblad and Brenntag Biosector, Denmark. The 13-valent pneumococcal conjugate vaccine (PCV13; Prevnar ${ }^{\circledR}$; Pfizer Inc.) as a PCV antigen model was commercially acquired. The PCV13 contains the capsular polysaccharide antigens of Streptococcus pneumoniae serotypes 1, 3, 4, 5, 6A, 6B, 7F, 9V, 14, 18C, 19A, 19F, and $23 \mathrm{~F}$ which individually conjugated to the nontoxic diphtheria toxin mutant $\mathrm{CRM}_{197}$.

2.2. Preparation of Adjuvant Nanoparticles. Chitosan nanoparticles were prepared by dissolving $0.2 \mathrm{~g}$ powdered chitosan in $0.5 \%$ acetic acid and mixing for 24 hours. After that, six drops of $10 \mathrm{M}$ sodium hydroxide were added until the $\mathrm{pH}$ reached to $4.6-4.8$. The obtained mixture was added with $0.5 \%$ sodium tripolyphosphate with a mole ratio of $3: 1$ for chitosan and sodium tripolyphosphate. The last step was homogenization of the mixture for 15 minutes with a stirring speed of $500 \mathrm{rpm}$ [10-12].

$35 \mathrm{mg}$ liposome nanoparticles were prepared by dissolving phosphatidylcholine into $3 \mathrm{~mL}$ chloroform and then by evaporation. Phosphate buffer saline ( $\mathrm{pH} 7.4)$ was added to modulate the thin layer of a lipid film [13]. Later on, this mixture was vortexed and sonicated for 15 minutes. The obtained liposomes were stored in a refrigerator until use.

Characterization of nanoparticles was carried out by a nanoparticle analyzer (Beckman Coulter Delsa) and transmission electron microscope (TEM) (JEM-1400, JEOL, Japan) for the mean particle size and the morphology of nanoparticles, respectively.

2.3. Mouse Immunization. The immunization study was approved by the Animal Care and Use Committee of PT. Bimana Indomedical, Bogor, Indonesia. Inbred 6-week-old female $\mathrm{BALB} / \mathrm{c}$ mice were maintained at the Animal Laboratory of PT. Bimana Indomedical, Bogor, Indonesia. Five mice per group were immunized intradermally with the PCV diluted in saline to $1: 10(100 \mu \mathrm{L}$ per mouse) [14] in mixture with adjuvants $(0.1-0.2 \mathrm{mg} / \mathrm{mL})$ : chitosan nanoparticles, liposome nanoparticles, and Quil-A adjuvant [15]. The Quil-A adjuvant which contains the water-extractable fraction of saponins from the South-American tree, Quillaja saponaria Molina, has been used to improve the immunogenicity of a synthetic carbohydrate-based vaccine [15]. Saline $(0.9 \%$ [wt/vol] $\mathrm{NaCl}$ in water) was used as a negative control. A booster of the PCV antigen diluted in saline to $1: 10(100 \mu \mathrm{L}$ per mouse) was given on day 35 without an adjuvant. Blood samples were taken one week after the booster immunization.

2.4. Measurement of Antibodies by ELISA. The enzymelinked immunosorbent assay (ELISA) was performed to measure the antibodies to pneumococcal type 14 polysaccharide (Pn14PS) antigen, one of the polysaccharide antigens included in the PCV, as described previously [16]. Briefly, serially diluted sera were incubated for $1 \mathrm{~h}$ at $37^{\circ} \mathrm{C}$ in flatbottom plates (Corning Inc., Corning, NY, USA), coated with $100 \mu \mathrm{L}$ of purified polysaccharide types 14 and $23(5 \mu \mathrm{g} / \mathrm{mL})$. After coating, the plates were blocked with 2\% gelatin (Sigma) and then washed and horseradish peroxidaseconjugated goat anti-mouse IgG (1:5000) (Silenus, Amrad Operations PTY LTD, Australia) or IgG subclass-specific antibodies (1:5000) (1 mg/mL; Abcam) were added and incubated for $1 \mathrm{~h}$ at $37^{\circ} \mathrm{C}$. A ready-to-use TMB (3,3',5, $5^{\prime}$-tetramethylbenzidine) with HRP (horseradish peroxidase) substrate (Life Technologies) was added to visualize the amount of bound peroxidase. The reaction was stopped by the addition of $0.5 \mathrm{M} \mathrm{H}_{2} \mathrm{SO}_{4}$. Optical density (OD) values were obtained with a microtiter plate spectrophotometer (Tecan Infinite F200, Switzerland) at $450 \mathrm{~nm}$. Antibody titers were expressed as the $\log 10$ of the dilution giving twice the OD obtained for control mice.

\section{Results}

3.1. Characterization of Nanoparticles. In this study, chitosan nanoparticles were prepared by the ionic gelation method with the interaction with the small anionic molecule tripolyphosphate (TPP). The TPP was used to prepare chitosan nanoparticles due to its characteristics being nontoxic, multivalent, and able to form gels through ionic interactions $[17,18]$. The formation of nanoparticles was indicated by the appearance of an opalescence, and it was confirmed by dynamic light scattering which indicated a size of $46.1 \mathrm{~nm}$ [19]. Liposome nanoparticles that were synthesized by the method of Kirby and Gregoriads resulted in nanoparticle size of $31.4 \mathrm{~nm}$. This result showed that the obtained liposomes reach the required particle size. Morphology of liposome and chitosan nanoparticles was investigated by using a TEM. The morphology of liposome nanoparticles appeared as unilamellar spherical shape (Figure 1(a)). Meanwhile, the morphology of chitosan nanoparticles is confirmed that the particles are small and individual in which they had a tendency to fuse. This fusion resulted in larger size particles due to the aggregation of these small particles (Figure 1(b)).

3.2. Effect of Adjuvants on Antibody Response and AntiPn14PS IgG Subclass Distribution. Mouse groups were immunized with PCV antigen with a series of adjuvants at day 0 , and booster injection was done with the same dose of the PCV without adjuvant codelivery at day 35 . The level of antibodies against pneumococcal type 14 polysaccharide antigens after the booster injection was determined by ELISA. The level of antibody against pneumococcal type 14 polysaccharide has the same pattern between the groups of mice with adjuvants and without adjuvant codelivery (Figure 2).

The diversification of anti-pneumococcal type 14 antibodies to other IgG subclasses was investigated after the booster injection. The PCV immunizations without an adjuvant mainly elicit IgG1 antibodies. Meanwhile, the mouse group with an adjuvant elicits the IgG subclass antibodies (Figure 3). The mouse group with the chitosan adjuvant evoked anti-Pn14PS IgG antibodies IgG1, IgG2a, IgG2b, and IgG3 so do the group of mice with the Quil-A adjuvant 


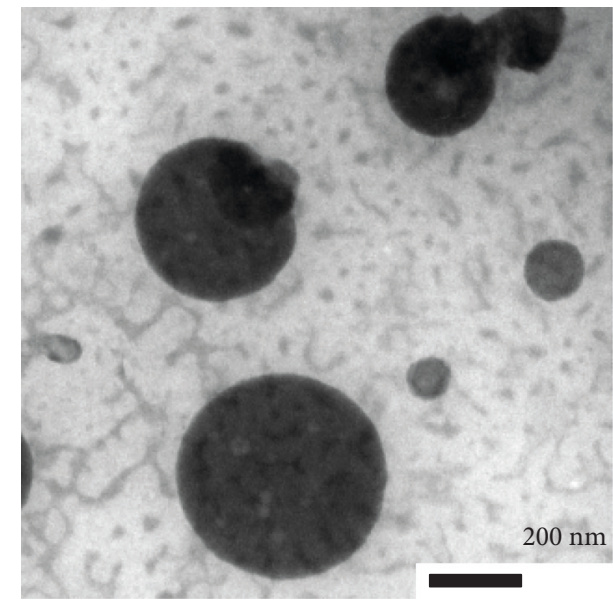

(a)

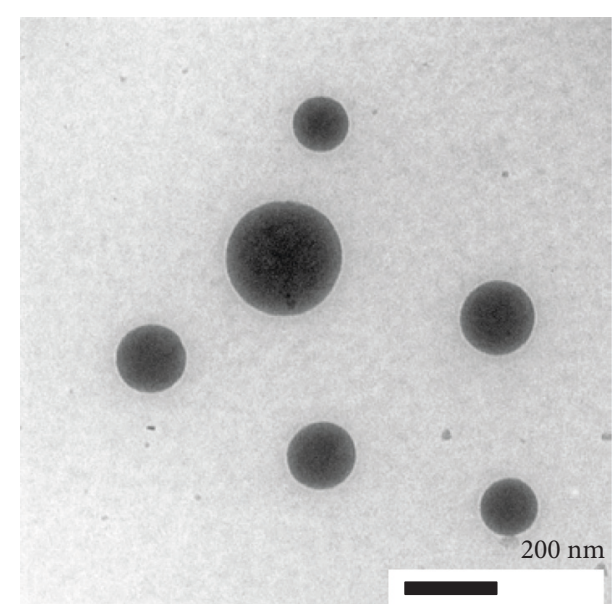

(b)

FIgURE 1: Morphology of nanoparticles (scale bar: $200 \mathrm{~nm}$ ): TEM image of chitosan nanoparticles (a) and TEM image of liposome nanoparticles (b).

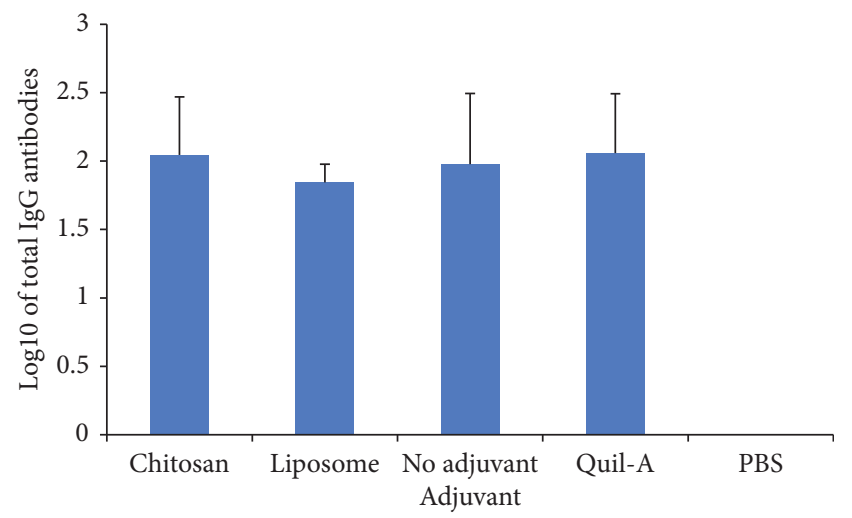

FIGURE 2: Total IgG antibody titers recognizing a series of pneumococcal polysaccharides of serotype 14. The group of mice $(n=5)$ was immunized with pneumococcal conjugate with a series of adjuvant coadministration at the primary injection. Sera were collected one week after the booster injection which was given without an adjuvant. Saline immunization served as the negative control. Antibody titers were expressed as the $\log 10$ of the dilution giving twice the absorbance value corrected by buffer.

as the positive control (Figure 3), while the liposome adjuvant could not show the diversification of IgG isotypes and mainly induces anti-Pn14PS IgG1.

\section{Discussion}

In this study, we observed that liposomes presented morphologically unilamellar spherical shape. Meanwhile, chitosan itself showed as the individual particle that has a tendency to fuse with each other creating larger size particles due to the aggregation inside it. The aggregation might be influenced by the conformation of the random coil in chitosan solution [20]. The obtained nanosize range as particles in those materials could make those to be used as a delivery system, here as an adjuvant. Many other TEM studies also indicated that the particle size, vesicle shape, and lamellarity of liposomes may be different due to the process of preparation [21].

In this study, the effect of adjuvant codelivery was shown by the diversification of anti-Pn14PS IgG subclasses. Previously, it was reported that the Quil-A adjuvant induced IgG diversification and elicited a broader anti-Pn14PS IgG subclass antibody spectrum [15]. We found that antibodies against type 14 polysaccharides between mouse groups with and without adjuvant codelivery were not significantly different. In this study, we also observed that PCV immunization with codelivery of chitosan nanoparticles significantly enhanced anti-Pn14PS IgG subclass responses. The IgG subclasses (IgG1, IgG2a, IgG2b, and IgG3) in mice immunized with the chitosan adjuvant show the same pattern as those in the Quil-A group as the positive control. Previously, Wen et al. [22] observed that mouse immunization with ovalbumin (OVA) and chitosan nanoparticles elicited a balanced Th1/Th2 immune response as indicated by the significant increases in IgG1, IgG2a, and IgG2b antibody subclasses.

We also found that liposome adjuvant immunization showed a low level of anti-Pn14PS IgG antibodies compared with other groups. The liposome adjuvant leads to an immune response dominated by IgG1. Previously, Korsholm et al. reported that one of the major drawbacks of liposomes as vaccine delivery vehicles has been their instability [23] Liposomes are versatile delivery systems for antigens, and they can carefully be customized towards desired immune profiles by combining them with immunostimulators and optimizing their composition, physicochemical properties, and antigen-loading mode [24].

Our data suggest that chitosan and liposome nanoparticles induced a Th2 (IgG2a, IgG2b, and IgG3) response rather than a Th1 (IgG1) response. In mice, immunoglobulin G1 (IgG1) is associated with a Th2 response, while a Th1 response is associated with the induction of IgG2a, IgG2b, and IgG3 antibodies [25]. 

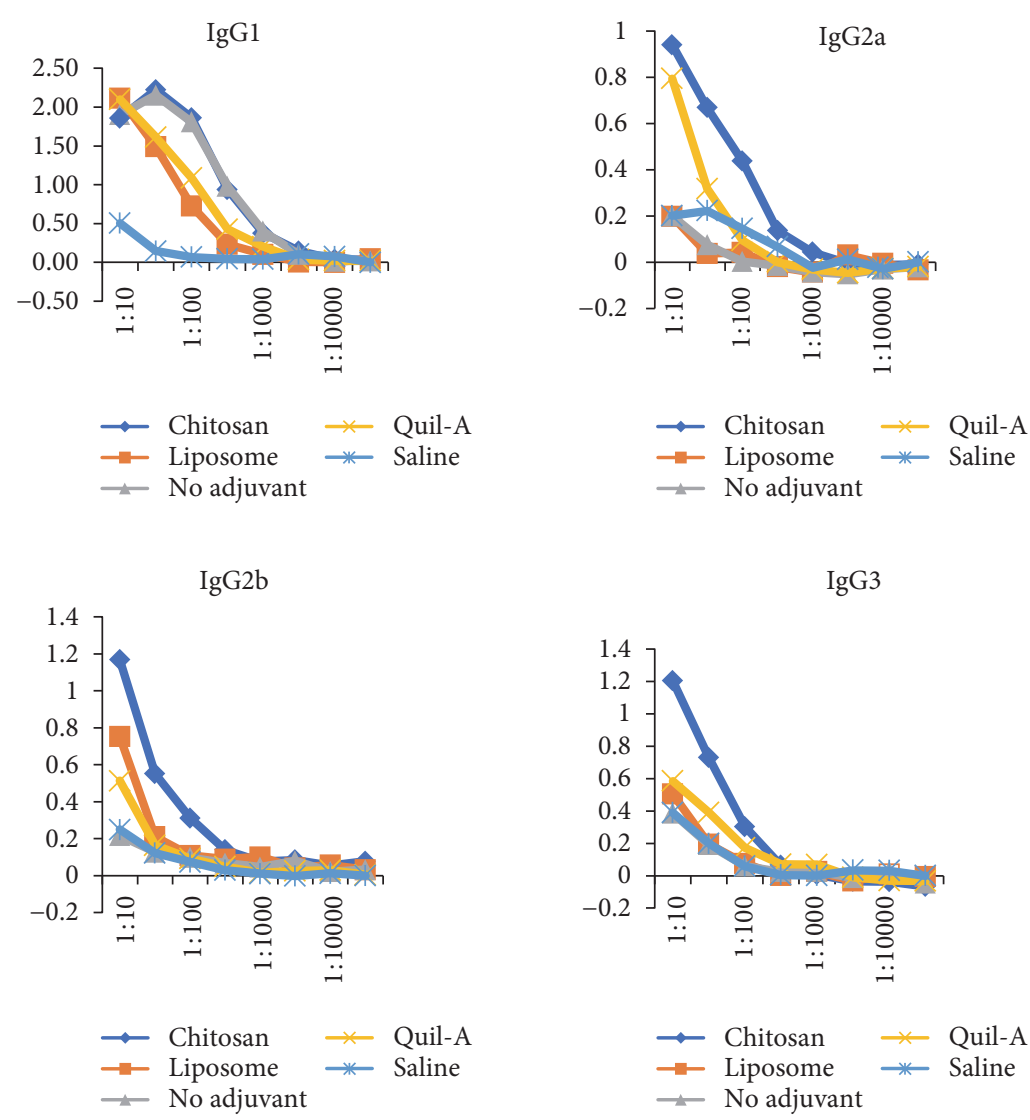

FIGURE 3: Anti-pneumococcal type 14 polysaccharide (anti-Pn14PS) IgG antibody subclass distribution. Mouse sera (ranging from 1:10 to 1:10000) were collected after the booster immunization. ELISA was performed to measure the anti-Pn14PS IgG antibody subclass distribution: IgG1, IgG2a, IgG2b, and IgG3. The level of antibodies is expressed as optical density (OD) at $450 \mathrm{~nm}$.

\section{Conclusion}

The chitosan and liposome nanoparticles as adjuvant codelivery in antibody production of mice were successfully synthesized. These nanoparticles have different shapes in particle formation, liposome nanoparticle with their unilamellar shape and chitosan nanoparticles in large shape due to the aggregation of small-size particles. Even though the nanometer size was achieved by both nanoparticles, the measurement of antibody production was different from both of them. In producing subclass antibody in a mouse model towards a commercial PCV, the codelivery of chitosan nanoparticles showed more effect compared to that of liposome nanoparticles. The obtained result due to the use of chitosan as adjuvant codelivery broadens the advantageous properties of chitosan as a biomaterial used in medicinal fields.

\section{Conflicts of Interest}

The authors declare that they have no conflicts of interest.

\section{Acknowledgments}

This study was supported by the grant of Competitive Program from Indonesian Institute of Sciences (LIPI, 2012) and Sinas Incentive Program from Dengue Vaccine
Consortium, Ministry of Research, Technology and Higher Education (2013). The authors also thank Yulianti Sampora, Hadidjah, and Fitriya N. A. Dewi for the technical and immunization assistance.

\section{References}

[1] A. Seth, F. K. Ritchie, N. Wibowo, L. H. L. Lua, and A. P. J. Middelberg, "Non-carrier nanoparticles adjuvant modular protein vaccine in a particle-dependent manner," PloS One, vol. 10, article e0117203, 2015.

[2] X. Li, M. Min, N. Du et al., "Chitin, chitosan, and glycated chitosan regulate immune responses: the novel adjuvants for cancer vaccine," Journal of Immunology Research, vol. 2013, Article ID 387023, 8 pages, 2013.

[3] L. Jiang, F. Qian, X. He et al., "Novel chitosan derivative nanoparticles enhance the immunogenicity of a DNA vaccine encoding hepatitis B virus core antigen in mice," The Journal of Gene Medicine, vol. 9, pp. 253-264, 2007.

[4] M. M. E. Temsahy, E. D. H. E. Kerdany, M. M. Eissa, T. I. Shalaby, I. M. Talaat, and N. M. F. H. Mogahed, "The effect of chitosan nanospheres on the immunogenicity of Toxoplasma lysate vaccine in mice," Journal of Parasitic Diseases, vol. 40, pp. 1-16, 2014.

[5] L. Sáenz, A. Neira-Carrillo, R. Paredes, M. Cortés, S. Bucarey, and J. L. Arias, "Chitosan formulations improve the immunogenicity of a GnRH-I peptide-based vaccine," International Journal of Pharmaceutics, vol. 369, pp. 64-71, 2009. 
[6] A. Akbarzadeh, R. Rezaei-Sadabady, S. Davaran et al., "Liposome: classification, preparation, and applications," Nanoscale Research Letters, vol. 8, pp. 1-9, 2013.

[7] A. Wagner, K. Vorauer-Uhl, A. Wagner, and K. Vorauer-Uhl, "Liposome technology for industrial purposes," Journal of Drug Delivery, vol. 2011, Article ID 591325, 9 pages, 2011.

[8] W. T. Al-Jamal and K. Kostarelos, "Liposome-nanoparticle hybrids for multimodal diagnostic and therapeutic applications," Nanomedicine, vol. 2, pp. 85-98, 2007.

[9] M. Henriksen-Lacey, D. Christensen, V. W. Bramwell et al., "Comparison of the depot effect and immunogenicity of liposomes based on dimethyldioctadecylammonium (DDA), 3 $\beta$-[N-(N',N'-dimethylaminoethane)carbomyl] cholesterol (DC-Chol), and 1,2-dioleoyl-3-trimethylammonium propane (DOTAP): prolonged liposome retention mediates stronger Th1 responses," Molecular Pharmaceutics, vol. 8, pp. 153-161, 2011.

[10] M. Bodnar, J. F. Hartmann, and J. Borbely, "Preparation and characterization of chitosan-based nanoparticles," Biomacromolecules, vol. 6, pp. 2521-2527, 2005.

[11] W. Fan, W. Yan, Z. Xu, and H. Ni, "Formation mechanism of monodisperse, low molecular weight chitosan nanoparticles by ionic gelation technique," Colloids and Surfaces. B, Biointerfaces, vol. 90, pp. 21-27, 2012.

[12] L. Qi, Z. Xu, X. Jiang, C. Hu, and X. Zou, "Preparation and antibacterial activity of chitosan nanoparticles," Carbohydrate Research, vol. 339, pp. 2693-2700, 2004.

[13] C. Kirby and G. Gregoriads, "Dehydration-rehydration vesicles (DRV); a new methods for high yield drug entrapment vesicles by trehalose during dehydration: retention of vesicle contents," Biochimica et Biophysica Acta, vol. 817, pp. 67-74, 1985.

[14] J. W. Rosch, A. R. Iverson, J. Humann et al., "A live-attenuated pneumococcal vaccine elicits CD4+ T-cell dependent class switching and provides serotype independent protection against acute otitis media," EMBO Molecular Medicine, vol. 6, pp. 141-154, 2014.

[15] D. Safari, H. A. T. Dekker, G. Rijkers, and H. Snippe, "Codelivery of adjuvants at the primary immunization site is essential for evoking a robust immune response to neoglycoconjugates," Vaccine, vol. 29, pp. 849-854, 2011.

[16] D. Safari, H. A. T. Dekker, J. A. F. Joosten et al., "Identification of the smallest structure capable of evoking opsonophagocytic antibodies against Streptococcus pneumoniae type 14," Infection and Immunity, vol. 76, pp. 4615-4623, 2008.

[17] A. M. De Campos, A. Sánchez, and M. J. Alonso, "Chitosan nanoparticles: a new vehicle for the improvement of the delivery of drugs to the ocular surface. Application to cyclosporin A," International Journal of Pharmaceutics, vol. 224, pp. 159-168, 2001.

[18] Y. Xu and Y. Du, "Effect of molecular structure of chitosan on protein delivery properties of chitosan nanoparticles," International Journal of Pharmaceutics, vol. 250, pp. 215-226, 2003.

[19] A. Rampino, M. Borgogna, P. Blasi, B. Bellich, and A. Cesàro, "Chitosan nanoparticles: preparation, size evolution and stability," International Journal of Pharmaceutics, vol. 455, pp. 219-228, 2013.

[20] M. X. Weinhold, J. C. M. Sauvageau, N. Keddig et al., "Strategy to improve the characterization of chitosan for sustainable biomedical applications: SAR guided multi-dimensional analysis," Green Chemistry, vol. 11, pp. 498-509, 2009.
[21] M. Brandl, D. Bachmann, M. Drechsler, and K. H. Bauer, "Liposome preparation using high-pressure homogenizers," in Liposomes Technology, G. Gregoriadis, Ed., vol. 1, pp. 4965, CRC Press Inc, Boca Raton, FL, 1993.

[22] Z.-S. Wen, Y.-L. Xu, X.-T. Zou, and Z.-R. Xu, “Chitosan nanoparticles act as an adjuvant to promote both Th1 and Th2 immune responses induced by ovalbumin in mice," Marine Drugs, vol. 9, pp. 1038-1055, 2011.

[23] K. Korsholm, P. Andersen, and D. Christensen, "Cationic liposomal vaccine adjuvants in animal challenge models: overview and current clinical status," Expert Review of Vaccines, vol. 11, pp. 561-577, 2012.

[24] S. T. Schmidt, C. Foged, K. S. Korsholm, T. Rades, and D. Christensen, "Liposome-based adjuvants for subunit vaccines: formulation strategies for subunit antigens and immunostimulators," Pharmaceutics, vol. 8, p. 7, 2016.

[25] T. Germann, M. Bongartz, H. Dlugonska et al., "Interleukin-12 profoundly up-regulates the synthesis of antigen-specific complement-fixing IgG2a, IgG2b and IgG3 antibody subclasses in vivo," European Journal of Immunology, vol. 25, pp. 823-829, 1995. 


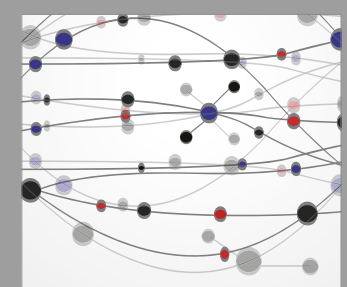

The Scientific World Journal
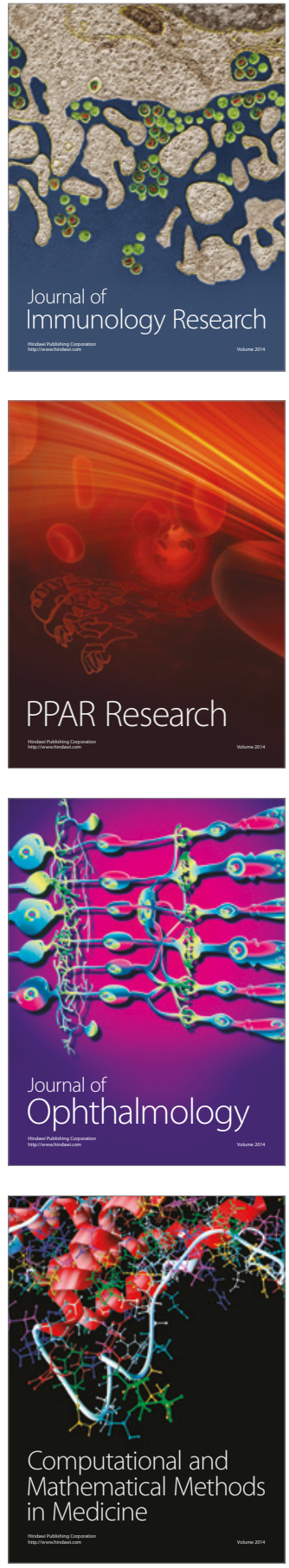

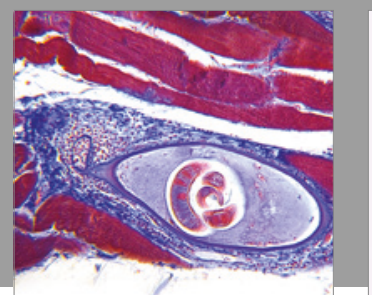

Gastroenterology Research and Practice
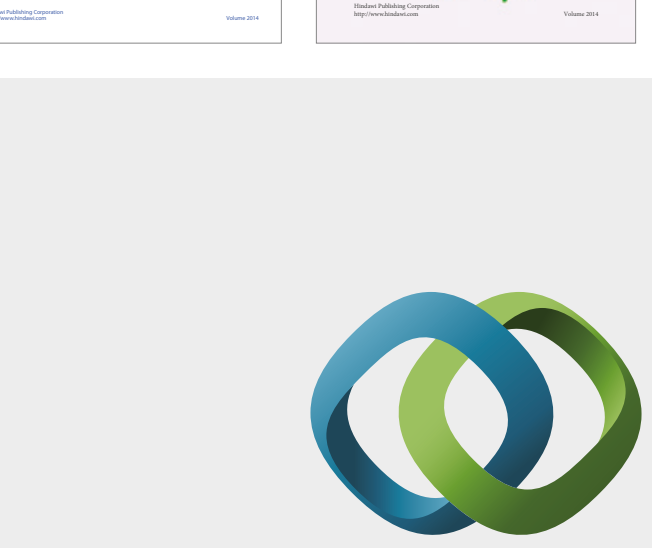

\section{Hindawi}

Submit your manuscripts at

https://www.hindawi.com
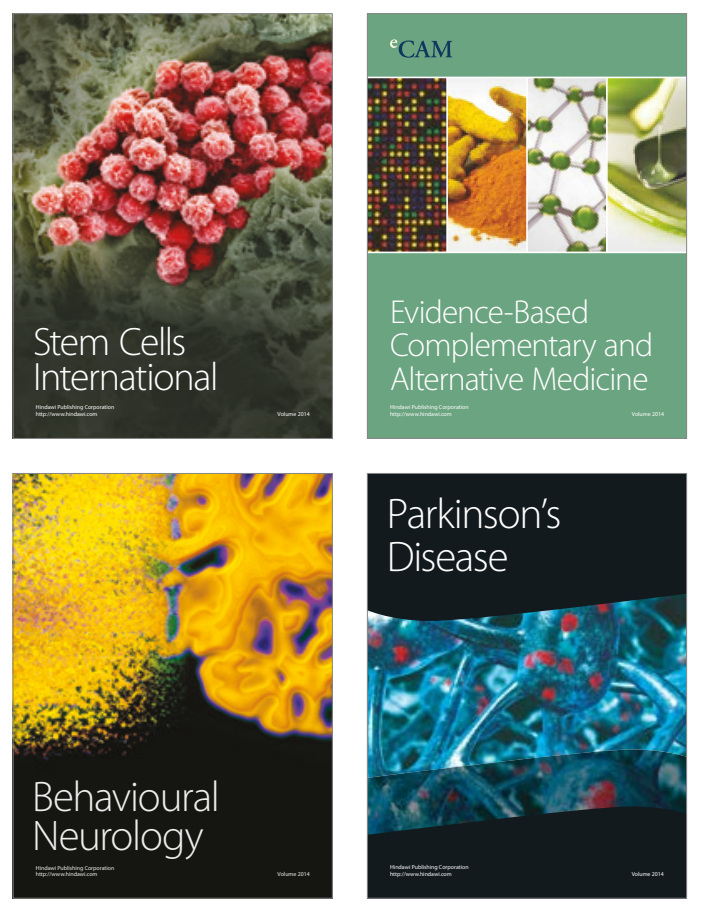
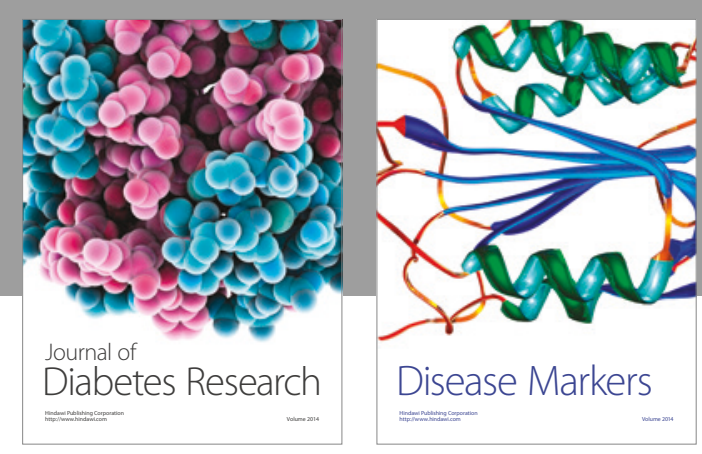

Disease Markers
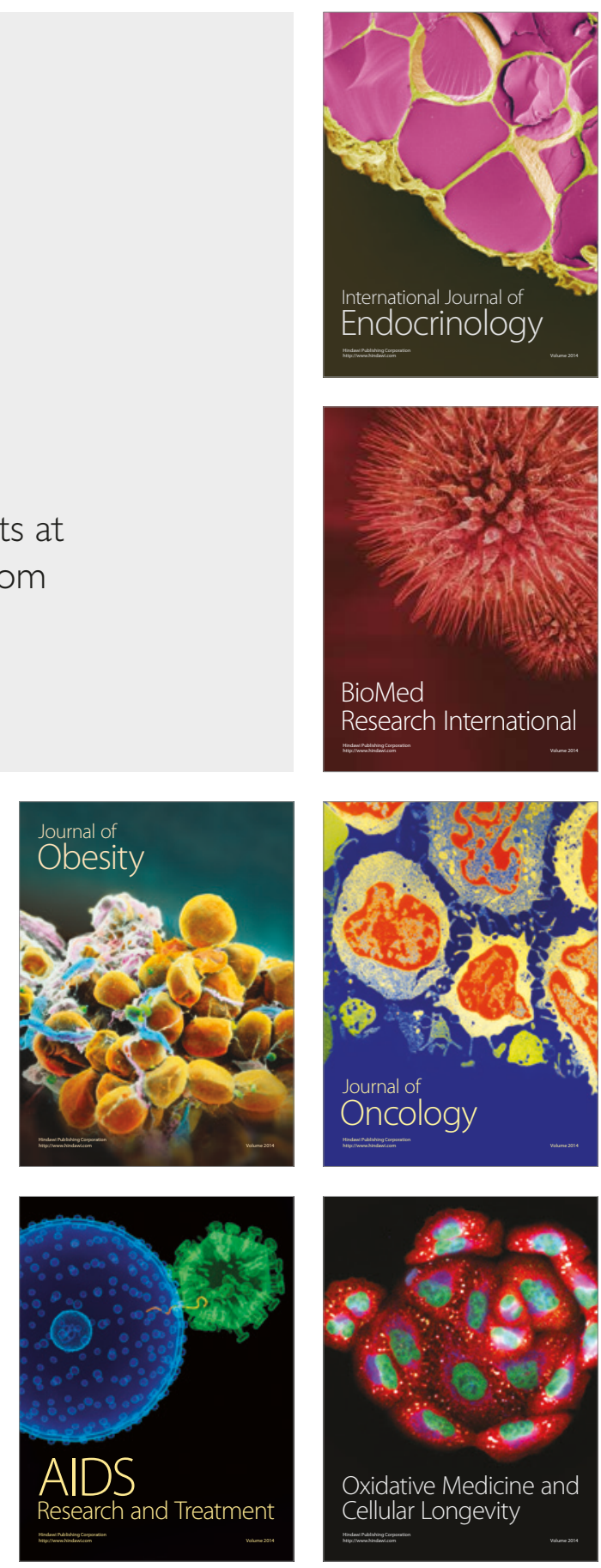